\title{
Prostate cancer: the great unknown
}

Approximately one out of three men over 50 years of age have a prostate cancer. Fortunately $90 \%$ of these tumours are not clinically significant and will never kill their host. Nevertheless 200,000 new prostate cancer cases were diagnosed in the USA in the year 2001, being the most frequently diagnosed cancer in men. In the same year 32,000 men died of prostate cancer, being the second cancer in mortality. In spite of the latter, the Federal Research Funding has spent significantly less money in prostate cancer research in comparison with other malignancies during the last decade. For instance, in 1996 only 80 millions US \$ were invested in prostate cancer research, compared to 600 millions US \$ invested in breast cancer research or 1,600 millions US \$ in AIDS. Taking in mind the relatively small budget directed for prostate cancer in USA, one can easily imagine the difficulties that investigators in other countries have to face. In spite of these drawbacks, important advances have been made in the knowledge of prostate carcinogenesis. And even more important is the fact that the time has come that new gene and cellular therapies are being tested in phase I and II studies. Doctor González et al summarize in their review the new treatment strategies that are being explored in prostate cancer. Phase I and II studies have not obtained dramatic responses, but have shown that gene therapy is possible in prostate cancer and can be used as biological sensitizer associated to classic adjuvant therapies, such as surgery or radiotherapy. They also have shown that gene and cellular therapies may be another way of treating disseminated tumours already resistant to androgen withdrawal.
The regulation of cell division by androgens is a key process in prostate cancer treatment. At least $80 \%$ of cancers initially respond to androgen withdrawal, which constitutes the main pillar of metastatic prostate cancer treatment. Regretfully the mean treatment response duration is only about 2 years. Thereafter almost all disseminated prostate cancers become resistant to hormone manipulation, progress and ultimately kill the patient. It would be ideal if we could avoid the tumours to become resistant to androgen blockage. In such a case prostate cancer would not be lethal and patients would die with their cancer but not of their cancer. Navarro et al review the way androgen receptors function in prostatic tumour cells and present the latest advances in this area. It has been show that the androgen receptor gene plays a significant role in prostate carcinogenesis and that it can regulate and change the susceptibility of a given subject to acquire a prostate tumour as well as its sensibility to respond to androgen deprivation. It is true that at the present time we still ignore the exact mechanism of how tumour cells turn into hormone resistant. Nevertheless I'm sure that new discoveries will give us the opportunity to avoid tumours to get hormone independent. At that point we will be able to improve the prognosis of our prostate cancer patients dramatically.

L. Martínez-Piñeiro Servicio de Urología. Hospital Universitario La Paz. Madrid. 